\title{
The sum theorem for maximal monotone operators in reflexive Banach spaces revisited
}

\author{
Mircea D. Voisei
}

Dedicated to Professor Gheorghe Moroşanu on the occasion of his 70th anniversary.

\begin{abstract}
The goal of this note is to present a new shorter proof for the maximal monotonicity of the Minkowski sum of two maximal monotone multi-valued operators defined in a reflexive Banach space under the classical interiority condition involving their domains.
\end{abstract}

Mathematics Subject Classification (2010): 47H05, 46N10.

Keywords: Maximal monotone operator, Minkowski sum.

\section{Preliminaries}

Recall the following sum rule for maximal monotone operators:

Theorem 1.1. (Rockafellar $[5$, Theorem 1 (a), p. 76]) Let $(X,\|\cdot\|)$ be a reflexive Banach space with topological dual $X^{*}$ and let $A, B: X \rightrightarrows X^{*}$ be multi-valued maximal monotone operators from $X$ to $X^{*}$. If $D(A) \cap \operatorname{int} D(B) \neq \emptyset$ then $A+B$ is maximal monotone.

Here $D(T):=\{x \in X \mid T(x) \neq \emptyset\}$ is the domain of $T: X \rightrightarrows X^{*}$, "int $S$ " denotes the topological interior of $S \subset X$, and $A+B: X \rightrightarrows X^{*}$ is the Minkowski sum of $A$ and $B$ defined by

$$
(A+B)(x):=A(x)+B(x):=\{y+v \mid y \in A(x), v \in B(x)\},
$$

for $x \in D(A+B):=D(A) \cap D(B)$.

The proof of $[5$, Theorem 1, p. 76] relies on the use of the duality mapping $J$ of $X$ and the (Minty's style) characterization of maximal monotone operators defined in reflexive Banach spaces. Similar arguments are used in the presence of an improved qualification constraint in a second proof of Theorem 1.1 (see [2, Corollary 3.5, p. 286]). A third proof of the main theorem involves the exact convolution of some specially constructed functions based on the Fitzpatrick functions of $A$ and $B$ (see [10, Corollary 
4, p. 1166]). A different proof of Theorem 1.1 is based on the dual-representability $A+B$ in the presence of the qualification constraint (see [8, Remark 1, p. 276]) and the fact that in a reflexive Banach space dual-representability is equivalent to maximal monotonicity (see e.g. [1, Theorem 3.1, p. 2381]). All the previously mentioned proofs make use of the duality mapping $J$ which is characteristic to a normed space.

Our proof relies on the normal cone, is based on full-range characterizations of maximal monotone operators with bounded domain, and uses the representability of sums of representable operators, but, avoids the use of $J$ or the norm. The following intermediary result, is the main ingredient of our argument.

Theorem 1.2. Let $X$ be a reflexive Banach space, let $T: X \rightrightarrows X^{*}$ be maximal monotone, and let $C \subset X$ be closed convex and bounded. If $D(T) \cap \operatorname{int} C \neq \emptyset$ then $T+N_{C}$ is maximal monotone.

Here $N_{C}$ denotes the normal cone to $C$ and is defined by $x^{*} \in N_{C}(x)$ if, for every $y \in C,\left\langle y-x, x^{*}\right\rangle \leq 0$. Here $\langle\cdot, \cdot\rangle$ denotes the coupling or duality product of $X \times X^{*}$ and is defined by

$$
c\left(x, x^{*}\right):=\left\langle x, x^{*}\right\rangle:=x^{*}(x), x \in X, x^{*} \in X^{*} .
$$

An element $z=\left(x, x^{*}\right) \in X \times X^{*}$ is monotonically related (m.r. for short) to $T$ if, for every $\left(a, a^{*}\right) \in \operatorname{Graph} T:=\left\{\left(u, u^{*}\right) \in X \times X^{*} \mid u \in D(T), u^{*} \in T(u)\right\}$, $\left\langle x-a, x^{*}-a^{*}\right\rangle \geq 0$.

Recall that a multi-valued operator $T: X \rightrightarrows X^{*}$ is

- monotone if, for every $x_{1}^{*} \in T\left(x_{1}\right), x_{2}^{*} \in T\left(x_{2}\right),\left\langle x_{1}-x_{2}, x_{1}^{*}-x_{2}^{*}\right\rangle \geq 0$.

- maximal monotone if every m.r. to $T$ element $z=\left(x, x^{*}\right) \in X \times X^{*}$ belongs to Graph $T$.

- representable if there is a proper convex $s_{X} \times w^{*}$-lower semicontinuous $h$ : $X \times X^{*} \rightarrow \mathbb{R} \cup\{+\infty\}$ such that $h \geq c$ and

$$
\text { Graph } T=[h=c]:=\left\{\left(x, x^{*}\right) \in X \times X^{*} \mid h\left(x, x^{*}\right)=\left\langle x, x^{*}\right\rangle\right\} .
$$

Here $s_{X}$ denotes the strong topology of $X$ and $w^{*}$ stands for the weak-star topology of $X^{*}$.

- $N I$ if $\varphi_{T} \geq c$, where $\varphi_{T}$ is the Fitzpatrick function of $T$ which is defined by

$$
\varphi_{T}\left(x, x^{*}\right):=\sup \left\{\left\langle x-a, a^{*}\right\rangle+\left\langle a, x^{*}\right\rangle \mid\left(a, a^{*}\right) \in \operatorname{Graph} T\right\},\left(x, x^{*}\right) \in X \times X^{*} \text {. }
$$

\section{Proofs of the main result}

Proof of Theorem 1.2. The operator $T+N_{C}$ is representable, which follows from the facts that $T, N_{C}$ are maximal monotone thus representable and $D(T) \cap \operatorname{int} C \neq \emptyset$ (see e.g. [6, Corollary 5.6, p. 470] or [7, Theorem 16, p. 818]).

We prove that $R\left(T+N_{C}\right)=X^{*}$ which implies that $T+N_{C}$ is of NI-type and so it is maximal monotone (see $[6$, Theorem 3.4, p. 465] or [8, Theorem 1 (ii), (7)]).

It suffices to prove that $0 \in R\left(T+N_{C}\right)$ otherwise we replace $T$ by $T-x^{*}$ for an arbitrary $x^{*} \in X^{*}$. 
Consider $F\left(x, x^{*}\right):=\varphi_{T}\left(x, x^{*}\right)+g\left(x, x^{*}\right)$, with $g\left(x, x^{*}\right):=\iota_{C}(x)+\sigma_{C}\left(-x^{*}\right)$, where $\iota_{C}(x)=0$, for $x \in C ; \iota_{C}(x)=+\infty$, otherwise, and $\sigma_{C}\left(x^{*}\right):=\sup _{x \in C}\left\langle x, x^{*}\right\rangle$, $x^{*} \in X^{*}$.

Then $F \geq 0$ due to $\varphi_{T}\left(x, x^{*}\right) \geq\left\langle x, x^{*}\right\rangle$ and $\iota_{C}(x)+\sigma_{C}\left(-x^{*}\right) \geq-\left\langle x, x^{*}\right\rangle$ (see f.i. [4]). Hence

$$
0 \leq \inf _{X \times X^{*}} F=-\left(\varphi_{T}+g\right)^{*}(0,0)=-\min _{\left(x, x^{*}\right) \in X \times X^{*}}\left\{\psi_{T}\left(x, x^{*}\right)+g^{*}\left(-x^{*},-x\right)\right\},
$$

because $C$ is bounded, $g$ is $s_{X} \times s_{X^{*}}$-continuous on $\operatorname{int} C \times X^{*}$, and $X$ is reflexive (see f.i. [9, Theorem 2.8.7, p. 126]), where $s_{X^{*}}$ is the strong topology of $X^{*}$. Here "min" denotes an infimum that is attained when finite,

$$
\psi_{T}\left(x, x^{*}\right)=\varphi_{T}^{*}\left(x^{*}, x\right),\left(x, x^{*}\right) \in X \times X^{*},
$$

the convex conjugation being taken with respect to the dual system

$$
\left(X \times X^{*}, X^{*} \times X^{* *}\right)
$$

and, for every $\left(x, x^{*}\right) \in X \times X^{*}, \psi_{T}\left(x, x^{*}\right) \geq\left\langle x, x^{*}\right\rangle$ because $T$ is monotone (see e.g. $[8,(12)])$.

From $g^{*}\left(x^{*}, x\right)=\iota_{C}(-x)+\sigma_{C}\left(x^{*}\right),\left(x, x^{*}\right) \in X \times X^{*}$ and (2.1) there exists $\left(\bar{x}, \bar{x}^{*}\right) \in X \times X^{*}$ such that $\psi_{T}\left(\bar{x}, \bar{x}^{*}\right)+\iota_{C}(\bar{x})+\sigma_{C}\left(-\bar{x}^{*}\right) \leq 0$ which implies that $\iota_{C}(\bar{x})+\sigma_{C}\left(-\bar{x}^{*}\right)=-\left\langle\bar{x}, \bar{x}^{*}\right\rangle$, i.e., $-\bar{x}^{*} \in N_{C}(\bar{x})$ and $\psi_{T}\left(\bar{x}, \bar{x}^{*}\right)=\left\langle\bar{x}, \bar{x}^{*}\right\rangle$, that is, $\bar{x}^{*} \in T(\bar{x})$ since $T$ is representable (see [8, Theorem 1, p. 270]).

Therefore $0 \in\left(T+N_{C}\right)\left(\bar{x}, \bar{x}^{*}\right)$ and so $0 \in R\left(T+N_{C}\right)$.

Proof of Theorem 1.1. First we prove that we can assume without loss of generality that $D(B)$ is bounded. Indeed, assume that the result is true for that case.

Let $z=\left(x, x^{*}\right)$ be m.r. to $A+B$. Take $C \subset X$ closed convex and bounded with $x \in \operatorname{int} C$ and $D(A) \cap \operatorname{int} D(B) \cap \operatorname{int} C \neq \emptyset$ e.g. $C:=\left[x_{0}, x\right]+U$, where

$$
\left[x_{0}, x\right]:=\left\{t x_{0}+(1-t) x \mid 0 \leq t \leq 1\right\}
$$

and $U$ is a closed convex bounded neighborhood of 0 , and $x_{0} \in D(A) \cap \operatorname{int} D(B)$. Note that $z$ is m.r. to $A+B+N_{C}=A+\left(B+N_{C}\right)$ which is maximal monotone since, according to Theorem 1.2, $B+N_{C}$ is maximal monotone, $D\left(B+N_{C}\right)$ is bounded, and $x_{0} \in D(A) \cap \operatorname{int} D\left(B+N_{C}\right) \neq \emptyset$. Hence $z \in \operatorname{Graph}\left(A+B+N_{C}\right)$ or $x^{*} \in(A+B)(x)$ because $N_{C}(x)=\{0\}$. Therefore $A+B$ is maximal monotone.

It remains to prove that, whenever $D(B)$ is bounded, $R(A+B)=X^{*}$ or sufficiently $0 \in R(A+B)$ (since $A+B$ is representable, see again [6, Corollary 5.6]).

Let

$$
F\left(x, x^{*}\right):=\varphi_{A}\left(x, x^{*}\right)+\varphi_{B}\left(x,-x^{*}\right), \quad g\left(x, x^{*}\right):=\varphi_{B}\left(x,-x^{*}\right), \quad\left(x, x^{*}\right) \in X \times X^{*} .
$$

Since $A, B$ are maximal monotone, for every $\left(x, x^{*}\right) \in X \times X^{*}$,

$$
\min \left\{\varphi_{A}\left(x, x^{*}\right), \varphi_{B}\left(x, x^{*}\right)\right\} \geq\left\langle x, x^{*}\right\rangle
$$

which imply $F \geq 0$ and so

$$
0 \leq \inf _{X \times X^{*}} F=-\left(\varphi_{A}+g\right)^{*}(0,0)=-\min _{\left(x, x^{*}\right) \in X \times X^{*}}\left\{\psi_{A}(x, x)+\psi_{B}\left(x,-x^{*}\right)\right\},
$$


because $D(B)$ bounded provides $D(B) \times X^{*} \subset \operatorname{dom} g, g$ is $s_{X} \times s_{X^{*}}$-continuous on int $D(B) \times X^{*}$, and $X$ is reflexive (see again [9, Theorem 2.8.7, p. 126]). More precisely, for every $\left(x, x^{*}\right) \in D(B) \times X^{*}$ there is $\bar{x}^{*} \in B(x)$ and so

$$
\begin{aligned}
\varphi_{B}\left(x, x^{*}\right) & :=\sup \left\{\left\langle x-b, b^{*}\right\rangle+\left\langle b, x^{*}\right\rangle \mid\left(b, b^{*}\right) \in \operatorname{Graph} B\right\} \\
& \leq \sup \left\{\left\langle x-b, \bar{x}^{*}\right\rangle+\left\langle b, x^{*}\right\rangle \mid\left(b, b^{*}\right) \in \operatorname{Graph} B\right\} \\
& \leq\left\langle x, \bar{x}^{*}\right\rangle+\left\|x^{*}-\bar{x}^{*}\right\| \sup _{b \in D(B)}\|b\|<+\infty .
\end{aligned}
$$

There exists $\left(\bar{x}, \bar{x}^{*}\right) \in X \times X^{*}$ such that $\psi_{A}\left(\bar{x}, \bar{x}^{*}\right)+\psi_{B}\left(\bar{x},-\bar{x}^{*}\right) \leq 0$ which implies that $\psi_{A}\left(\bar{x}, \bar{x}^{*}\right)=\left\langle\bar{x}, \bar{x}^{*}\right\rangle, \psi_{B}\left(\bar{x},-\bar{x}^{*}\right)=-\left\langle\bar{x}, \bar{x}^{*}\right\rangle$, i.e., $\bar{x}^{*} \in A(\bar{x})$ and $-\bar{x}^{*} \in B(\bar{x})$ from which $0 \in R(A+B)$.

Remark 2.1. Theorem 1.2 still holds if we replace the assumption $C$ bounded with $D(T)$ bounded. In this case an alternate proof of Theorem 1.1 can be performed with $A+N_{C}$ instead of $A$ and a similar argument as in the current proof.

\section{References}

[1] Burachik, R.S., Svaiter, B.F., Maximal monotonicity, conjugation and the duality product, Proc. Amer. Math. Soc., 131(2003), no. 3, 2379-2383 (electronic)

[2] Chu, L.-J., On the sum of monotone operators, Michigan Math. J., 43(1996), no. 2, 273-289.

[3] Debrunner, H., Flor, P., Ein Erweiterungssatz für monotone Mengen, Arch. Math., 15(1964), 445-447.

[4] Fitzpatrick, S., Representing monotone operators by convex functions, In" "Workshop/Miniconference on Functional Analysis and Optimization" (Canberra, 1988), vol. 20 of Proc. Centre Math. Anal. Austral. Nat. Univ., Austral. Nat. Univ., Canberra, 1988, 59-65.

[5] Rockafellar, R.T., On the maximality of sums of nonlinear monotone operators, Trans. Amer. Math. Soc., 149(1970), 75-88.

[6] Voisei, M.D., The sum and chain rules for maximal monotone operators, Set-Valued Anal., 16(2008), no. 4, 461-476.

[7] Voisei, M.D., Maximal monotone normal cones in locally convex spaces, J. Math. Anal. Appl., 476(2019), no. 2, 811-818.

[8] Voisei, M.D., Zălinescu, C., Maximal monotonicity criteria for the composition and the sum under weak interiority conditions, Math. Program., 123(2010), no. 1, Ser. B, 265283.

[9] Zălinescu, C., Convex Analysis in General Vector Spaces, World Scientific Publishing Co. Inc., River Edge, NJ, 2002.

[10] Zălinescu, C., A new proof of the maximal monotonicity of the sum using the Fitzpatrick function, In: Variational analysis and applications, vol. 79 of Nonconvex Optim. Appl., Springer, New York, 2005. 1159-1172.

Mircea D. Voisei

Baltimore, MD-21252, U.S.A.

e-mail: mdvoisei@yahoo.com 